\title{
ON FUNCTION SPECTRA
}

\author{
HAROLD M. HASTINGS
}

\begin{abstract}
We construct an explicit internal mapping functor for the homotopy category of CW spectra; this, together with our earlier smash product, yields a symmetric monoidal closed category.
\end{abstract}

Introduction. Boardman [2], [9], Adams [1], and the author [5] have defined smash products on the homotopy category of $\mathrm{CW}$ spectra which yield a symmetric monoidal category [4]. Further, Boardman and Heller [6] have used Brown's theorem [6, Theorem 12.2] to define an adjoint internal mapping functor, and thus show that such a category is closed. Our construction avoids Brown's theorem.

We thank Alex Heller, William Massey, and John C. Moore for helpful discussions.

1. CW spectra. We gave a definition equivalent to the usual definition (e.g., [1], [2]) by applying the Adams completion ([1], [6]) to the following category (in [5]):

Definition 1. A (CW) prespectrum $\boldsymbol{X}$ consists of a sequence of pointed CW complexes $\left\{\boldsymbol{X}_{n} \mid n \geqq 0\right\}$, together with cellular inclusions $\boldsymbol{X}_{n} \wedge S^{4} \rightarrow \boldsymbol{X}_{n+1}$. Ps is the category of prespectra and strict (continuous, pointed) maps.

Here $S^{0}=\partial I, S^{1}=I / S^{0}, S^{n}=S^{1} \wedge \cdots \wedge S^{1}$ for $n>1$, and $S^{n}=$ pt otherwise. Denote prespectra with boldface type.

Call a cellular inclusion $\boldsymbol{X}^{\prime} \subset \boldsymbol{X}$ cofinal [1] if the (quadruple) suspensions of each cell of each $\boldsymbol{X}_{n}$ are eventually in $\boldsymbol{X}^{\prime}$.

Definition 2. Objects of Ad are prespectra, and

$$
\operatorname{Ad}(\boldsymbol{X}, \boldsymbol{Y})=\operatorname{colim}\left\{\operatorname{Ps}\left(\boldsymbol{X}^{\prime}, \boldsymbol{Y}\right) \mid \boldsymbol{X}^{\prime} \text { cofinal in } \boldsymbol{X}\right\} \text {. }
$$

For Theorem 5 below we shall need the following equivalent definition of $\operatorname{Ad}(\boldsymbol{X}, \boldsymbol{Y})$ due to Boardman and Heller.

Call a prespectrum $\boldsymbol{X}$ finite if, for sufficiently large $n, \boldsymbol{X}_{n}$ is a finite complex and $\boldsymbol{X}_{n+1}=\boldsymbol{X}_{n} \wedge S^{4}$. Use (1) to define $\operatorname{Ad}(\boldsymbol{X}, \boldsymbol{Y})$ for finite $\boldsymbol{X}$.

Received by the editors February 22, 1973.

AMS (MOS) subject classifications (1970). Primary 55B20; Secondary 18D15, $55 \mathrm{~J} 99$.

Key words and phrases. Adams category, Boardman category, $h-c$ category, generalized cohomology theory, the Brown theorem, realization of singular complex.

(c) American Mathematical Society 1974 
Extend this by regarding an arbitrary prespectrum $X$ as the colimit of its finite subspectra.

Define a smash product $\wedge: \mathrm{CW}, \mathrm{Ad} \rightarrow \mathrm{Ad}$ by $(K \wedge X)_{n}=K \wedge X_{n}$; define homotopy with the cylinder functor $I^{*} \wedge$ ?; and let $\mathrm{Ht}(\mathrm{Ad})$ be the resulting homotopy category. See [1] or [5] for details.

We shall also need the category Ws of weak prespectra, which is defined as follows. In Definition 1, replace "CW complex" by "compactly generated space" [8], and replace "cellular inclusion" by "continuous map."

Observe Ps $\subset$ Ws. Extend Ad( , ) to a functor on $A d \times$ Ws.

2. Construction of the internal mapping functor MAP. Let $\boldsymbol{X}$ and $\boldsymbol{Y}$ be prespectra. As a first approximation, define a weak prespectrum $\operatorname{Map}(\boldsymbol{X}, \boldsymbol{Y})$ as follows. Let $\boldsymbol{S}^{-4 n}$ be the $-4 n$-sphere prespectrum: $\left(S^{-4 n}\right)_{i}=S^{4 i-4 n}$; the required inclusions are induced by $S^{4 i-4 n} \wedge S^{4} \cong$ $S^{4 i-4 n+4}$ for $i+n \geqq 0$. Choose a representative smash product on $\mathrm{Ht}(\mathrm{Ad})$ $[5, \S 3]$; as in [5], the mapping functor will be independent of this choice. Let $\operatorname{Map}(\boldsymbol{X}, \boldsymbol{Y})_{n}=\operatorname{Ad}\left(\boldsymbol{S}^{-4 n} \wedge \boldsymbol{X}, \boldsymbol{Y}\right)$, with the topology induced from the compactly generated function spaces [8] $\operatorname{Map}\left(\left(\boldsymbol{S}^{-4 n} \wedge \boldsymbol{X}\right)_{i}, \boldsymbol{Y}_{i}\right)$. The maps $S^{4} \wedge S^{-4 n-4} \rightarrow S^{-4 n}$ induced the required maps

$$
\operatorname{Map}(X, Y)_{n} \wedge S^{4} \rightarrow \operatorname{Map}(X, Y)_{n+1} .
$$

Remarks 3. To construct an adjoint to the Boardman-Adams smash product, replace " $S^{-4 n}$ " by " $S^{-n}$," and " $S^{4}$ " by " $S$ " in the above construction.

Definition 4. Let $\operatorname{MAP}(\boldsymbol{X}, \boldsymbol{Y})$ be the (degreewise) realization of the singular complex [7] of the telescope [5] of $\operatorname{Map}(X, Y)$.

Extend MAP to a functor $\mathrm{Ht}(\mathrm{Ad}) \times \mathrm{Ht}(\mathrm{Ad}) \rightarrow \mathrm{Ht}(\mathrm{Ad})$.

3. The closed structure. We show that MAP is the required internal mapping functor.

THEOREM 5. $\operatorname{Ht}(\operatorname{Ad})(X \wedge Y, Z) \cong H t(\operatorname{Ad})(X, \operatorname{MAP}(Y, Z))$.

Proof. First observe that, for finite $\boldsymbol{X}$ and $\boldsymbol{Y}$,

$$
\operatorname{Ad}(\boldsymbol{X} \wedge \boldsymbol{Y}, \boldsymbol{Z}) \cong \operatorname{Ad}(\boldsymbol{X}, \operatorname{Map}(\boldsymbol{Y}, \boldsymbol{Z})) .
$$

By taking colimits, and using the Boardman-Heller completion ( $(1)$, we obtain (2), and hence its analogue in $\mathrm{Ht}(\mathrm{Ad})$, for arbitrary prespectra. Finally, there are natural weak homotopy equivalences $\operatorname{MAP}(\boldsymbol{Y}, \boldsymbol{Z}) \rightarrow$ $\operatorname{Map}(\boldsymbol{Y}, \boldsymbol{Z})$; thus

$$
\operatorname{Ht}(\operatorname{Ad})(X, \operatorname{Map}(Y, Z)) \cong \operatorname{Ht}(\operatorname{Ad})(X, \operatorname{MAP}(Y, Z))
$$

by [1, Theorem 3.4$]$. The conclusion follows. 
Corollary 6. $\pi_{0} \operatorname{MAP}(,) \cong \operatorname{Ht}(\operatorname{Ad})(, \quad)$.

This follows from the isomorphism $\pi_{0} \cong \operatorname{Ht}(\operatorname{Ad})\left(S^{0},\right)$, which also shows that $\mathrm{Ht}(\mathrm{Ad})$ is normalized [4, p. 491].

The remaining coherence conditions for a closed category [4, p. 491, Theorem 5.5] are easily verified; their precise statement and proof are omitted.

REMARKS 7. For a weak prespectrum $\boldsymbol{W}, \pi_{-*} \operatorname{MAP}(?, \boldsymbol{W})$ is a generalized cohomology theory, compare the dual relationship between spectra and generalized homology theories ([9], [1]). An internal mapping functor on the homotopy category of pointed $\mathrm{CW}$ complexes may be constructed analogously with Definition 3; its existence follows from Brown's theorem [3].

\section{REFERENCES}

1. J. F. Adams, Stable homotopy and generalized homology, notes by R. Ming, Univ. of Chicago Math Lecture Notes, 1971.

2. J. M. Boardman, Stable homotopy theory, Univ. of Warwick, 1965.

3. E. H. Brown, Jr., Cohomology theories, Ann. of Math. (2) 75 (1962), 467-484; 78 (1963), 201. MR 25 \#1551; 27 \#749.

4. S. Eilenberg and G. M. Kelly, Closed categories, Proc. Conf. Categorical Algebra (La Jolla, Calif., 1965), Springer, New York, 1966, pp. 421-562. MR 37 \#1432.

5. H. M. Hastings, A smash product for spectra, Bull, Amer. Math. Soc. 79 (1973), 944-949.

6. A. Heller, Completions in abstract homotopy theory, Trans. Amer. Math. Soc. 147 (1970), 573-602. MR 41 \#2676.

7. D. M. Kan, Semisimplicial spectra, Illinois J. Math. 7 (1963), 463-478. MR 27 2986.

8. N. E. Steenrod, A convenient category of topological spaces, Michigan Math. J. 14 (1967), 133-152. MR 35 \#970.

9. R. Vogt, Boardman's stable homotopy category, Lecture Note Series, no. 21, Aarhus Univ., Aarhus, 1970. MR 43 \#1187.

10. G. W. Whitehead, Generalized homology theories, Trans. Amer. Math. Soc. 102 (1962), 227-283. MR 25 \#573.

Mathematics Department, Hofstra University, Hempstead, New York 11550 